\title{
Optimization of the contrast in polarimetric scalar images
}

\author{
François Goudail* and Arnaud Bénière \\ Laboratoire Charles Fabry de l'Institut d'Optique, CNRS, Université Paris-Sud, \\ Campus Polytechnique, RD 128, 91127 Palaiseau, France \\ *Corresponding author: francois.goudail@institutoptique.fr
}

Received February 23, 2009; revised April 3, 2009; accepted April 3, 2009; posted April 8, 2009 (Doc. ID 107926); published April 30, 2009

\begin{abstract}
We consider active polarimetric imaging systems that illuminate a scene with an incident polarization state and project the backscattered light on another polarization state in order to produce a scalar intensity image. We present and analyze a method for determining the configuration of illumination and analysis polarization states that maximizes the observed contrast between a target and the background when the scene is partially depolarizing and in the presence of additive Gaussian detection noise. (C) 2009 Optical Society of America
\end{abstract}

OCIS codes: $260.5430,030.4280$.

We consider active polarimetric imaging systems that illuminate a scene with an incident polarization state defined by its Stokes vector $\vec{S}$ and project the backscattered light on another polarization state, defined by its Stokes vector $\vec{T}$ in order to produce a scalar intensity image (see Fig. 1). This type of imaging provides useful information about the scene and makes it possible to discriminate objects that have different polarization scattering properties. In this Letter, we seek to optimize the contrast between two regions in the scene that have different polarization properties, such as a target of interest and a background. The degrees of freedom available to perform this optimization are the polarization states $\vec{S}$ and $\vec{T}$. This issue is crucial in remote sensing and biomedical applications where only a single scalar image can be recorded. It has been addressed for radar signals perturbed by speckle noise $[1,2]$ and for lidar detection of immersed targets by using different optimization approaches [3].

Our purpose is to discriminate two regions $a$ and $b$ whose polarimetric characteristics are described by their Mueller matrices $M_{a}$ and $M_{b}$. We use the Mueller formalism [4], since we are interested in remote sensing or biomedical applications, where scenes are often highly depolarizing and the Jones formalism [4] is inadequate. The scene is illuminated with purely polarized light that can have any Stokes vector $\vec{S}$ on the Poincare sphere (see Fig. 1) and is produced by a polarization state generator. The Stokes vector scattered by region $a(b)$ is $\vec{S}_{a}=M_{a} \vec{S}\left(\vec{S}_{b}=M_{b} \vec{S}\right)$. The light backscattered by the scene is analyzed with a generalized polarizer whose eigenstate is the Stokes vector $\vec{T}$. The measured intensities that correspond to region $u=a$ or $b$ are [4]

$$
I_{u}=\frac{1}{2}\left(\vec{T}^{T} M_{u} \vec{S}\right),
$$

where the superscript $T$ denotes matrix transposition.

The appropriate expression of the contrast in an image depends on the statistical properties of the noise that perturbs it [5]. In this Letter, we will as- sume that the noise is additive and Gaussian, since it is a simple model, generally adequate for detection noise. In the presence of additive Gaussian noise with variance $\sigma^{2}$, an adequate expression of the contrast between regions $a$ and $b$ is [5]

$$
\mathcal{C}(\vec{S}, \vec{T})=\frac{1}{\sigma^{2}}\left(I_{a}-I_{b}\right)^{2}=\frac{1}{4 \sigma^{2}}\left(\vec{T}^{T} D \vec{S}\right)^{2},
$$

where $D=\left(M_{a}-M_{b}\right)$.

Our objective is to determine the Stokes vectors $\vec{S}$ and $\vec{T}$ that maximize this contrast. Let $f(x)$ be a scalar function of a parameter $x$, which may be vectorial. One has $\max _{x}[f(x)]^{2}=\left[\max _{x}|f(x)|\right]^{2}$. To maximize $\mathcal{C}(\vec{S}, \vec{T})$, it is thus sufficient to determine the maximum and the minimum of the function

$$
F(\vec{S}, \vec{T})=\vec{T}^{T} D \vec{S}
$$

To simplify equations, we will assume that $\vec{S}$ has unit intensity and use the following notation:

$$
\vec{S}=\left(\begin{array}{l}
1 \\
\vec{s}
\end{array}\right), \quad \vec{T}=\left(\begin{array}{l}
1 \\
\vec{t}
\end{array}\right), \quad D=\left[\begin{array}{cc}
D_{00} & \vec{m}^{T} \\
\vec{n} & \widetilde{D}
\end{array}\right],
$$

where $\widetilde{D}$ is a $3 \times 3$ matrix and $\vec{s}$ and $\vec{t}$ are threedimensional unit norm vectors. The function in Eq. (3) can thus be written as

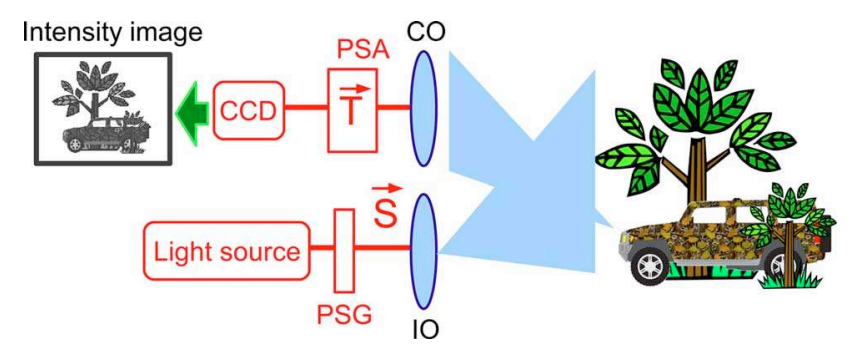

Fig. 1. (Color online) Principle of active scalar polarimetric imaging. PSG, polarization state generator; PSA, polarization state analyzer; IO, illumination optics; $\mathrm{CO}$, collection optics. 


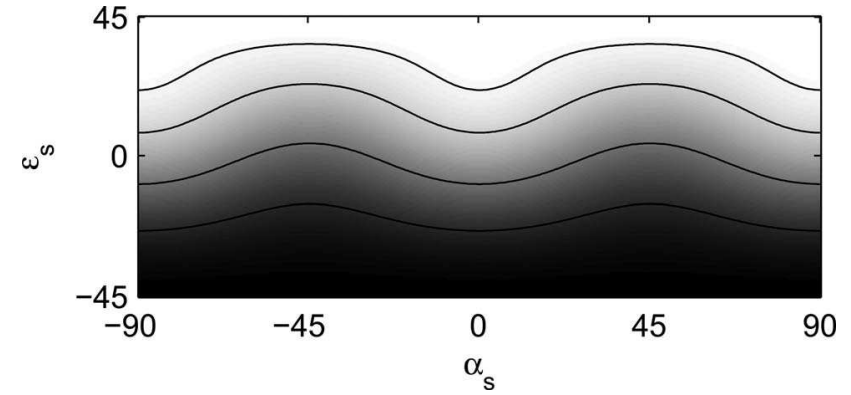

Fig. 2. Reduced contrast function $G^{\prime}\left(\alpha_{s}, \varepsilon_{s}\right)$ for $\gamma=0.096$.

$$
F(\vec{s}, \vec{t})=D_{00}+\vec{s}^{T} \vec{m}+\vec{t}^{T} \vec{n}+\vec{t}^{T} \widetilde{D} \vec{s}
$$

If the input light has intensity $I_{0}$, the contrast $\mathcal{C}(\vec{s}, \vec{t})=\left[I_{0} /(2 \sigma) F(\vec{s}, \vec{t})\right]^{2}$ is proportional to the square of $F(\vec{s}, \vec{t})$.

To simplify computations, let us consider the singular value decomposition [6] of matrix $\widetilde{D}$, which is in general not symmetric:

$$
\widetilde{D}=Y \Lambda X^{T}
$$

where $\Lambda$ is a diagonal matrix whose values $\lambda_{i}$ are nonnegative and $X$ and $Y$ are orthogonal matrices. Let us also define the following parameters:

$$
\vec{u}=X^{T} \vec{s}, \quad \vec{v}=Y^{T} \vec{t}, \quad \vec{p}=X^{T} \vec{m}, \quad \vec{q}=Y^{T} \vec{n} .
$$

After some computation, the function in Eq. (5) can be written as

$$
F(\vec{u}, \vec{v})=D_{00}+\vec{p}^{T} \vec{u}+\vec{v}^{T}(\vec{q}+\Lambda \vec{u}) .
$$

Finding the minimum and the maximum of $F(\vec{u}, \vec{v})$ under the constraint that $\|\vec{u}\|^{2}=\|\vec{v}\|^{2}=1$ is an optimization problem with four parameters. We will show in the following that it can be reduced to a twoparameter optimization problem, which results in a significant acceleration of the computations and also brings physical insight into the solution of the problem. Let us first consider that $\vec{u}$ is fixed and determine the vectors $\vec{v}$ that correspond to extrema of $F(\vec{u}, \vec{v})$. This is simply done by maximizing the scalar product $\vec{v}^{+}(\vec{q}+\Lambda \vec{u})$. Taking the constraint $\|\vec{v}\|=1$ into account, one obtains two solutions:

$$
\vec{v}_{+}=\frac{\vec{q}+\Lambda \vec{u}}{\|\vec{q}+\Lambda \vec{u}\|}, \quad \vec{v}_{-}=-\vec{v}_{+} .
$$

Simple computations show that these two solutions lead to the following values of $F$ :

$$
\begin{aligned}
& F\left(\vec{u}, \vec{v}_{+}\right)=A(\vec{u})+B(\vec{u}), \\
& F\left(\vec{u}, \vec{v}_{-}\right)=A(\vec{u})-B(\vec{u}),
\end{aligned}
$$

with

$$
A(\vec{u})=D_{00}+\vec{p}^{T} \vec{u}, \quad B(\vec{u})=\|\vec{q}+\Lambda \vec{u}\| .
$$

Thus using the fact that $B>0$, one obtains the reduced contrast function

$$
G(\vec{u})=\max _{\vec{v}}\left\{[F(\vec{u}, \vec{v})]^{2}\right\}=[|A(\vec{u})|+B(\vec{u})]^{2} .
$$

The value of $\vec{v}$ that leads to the maximum is $\vec{v}_{+}$if $A(\vec{u})>0$ and $\vec{v}_{-}$if $A(\vec{u})<0$. If $A(\vec{u})=0$, both vectors lead to a maximum. In summary, the proposed algorithm is the following:

- Determine $\vec{u}_{\text {opt }}=\arg \max _{\vec{u}}[G(\vec{u})]$ with numerical optimization software.

- The optimal value of $\vec{v}$ is

$$
\vec{v}_{\text {opt }}=\operatorname{sign}\left[A\left(\vec{u}_{\text {opt }}\right)\right] \frac{\vec{q}+\Lambda \vec{u}_{\text {opt }}}{\left\|\vec{q}+\Lambda \vec{u}_{\text {opt }}\right\|},
$$

where $\operatorname{sign}(x)=1$ if $x>0$ and -1 otherwise, $A(\vec{u})$ is defined in Eq. (12). If $A\left(\vec{u}_{\text {opt }}\right)=0$, plus and minus signs both correspond to an optimum.

- The optimal input polarization state is $\vec{s}_{\text {opt }}$ $=X \vec{u}_{\text {opt }}$, and the optimal analysis state is $\vec{t}_{\text {opt }}=Y \vec{v}_{\text {opt }}$, where $X$ and $Y$ are defined in Eq. (6).

Let us consider two particular cases where the solution is a closed-form one that also brings physical insight. The first one corresponds to $\vec{m}=\vec{n}=0$, which happens when regions $a$ and $b$ have identical polarizance and diattenuation vectors [4]. In this case, it is noticed in Eq. (8), that if $(\vec{u}, \vec{v})$ is a solution then $(-\vec{u},-\vec{v})$ also is. The function to maximize is $\left|D_{00}\right|$ $+\|\Lambda \vec{u}\|$. The solution is obvious: if $\lambda_{1}$ is the greatest value of the diagonal matrix $\Lambda, \vec{u}_{\text {opt }}=( \pm 1,0,0)^{T}$. Consequently, $\vec{s}_{\text {opt }}$ is proportional to the right-side singular vector associated with the largest singular value of $\widetilde{D}$. The value of $\vec{v}_{\text {opt }}$ depends on the sign of $D_{00}$. If $D_{00}>0$, that is, if the unpolarized intensity reflectivity of region $a$ is larger than that of region $b$, then Eq. (14) leads to $\vec{v}_{\text {opt }}=\vec{u}_{\text {opt }}$, that is, $\vec{t}_{\text {opt }}$ is the left-side singular vector associated to the largest singular value. On the other hand, if $D_{00}<0$, then $\vec{v}_{\text {opt }}=-\vec{u}_{\text {opt }}$, and thus $\vec{t}_{\text {opt }}$ is opposite to what it was in the previous case. This leads to an interesting interpretation: the sign of $\vec{t}_{\text {opt }}$ is such that the intensity contribution to the contrast, that is, $D_{00}$, has the same sign as the polarimetric contribution to the contrast, that is, $\vec{t}_{\text {opt }}^{T} \widetilde{D} \vec{s}_{\text {opt }}$, so that the absolute value of the sum of both

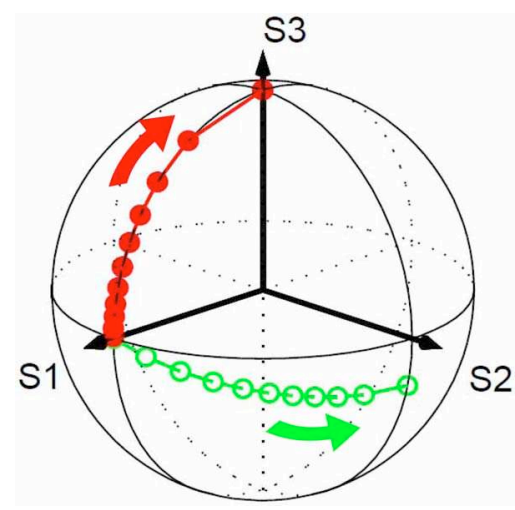

Fig. 3. (Color online) Representation of $\vec{s}_{\text {opt }}(\bullet)$ and $\vec{t}_{\text {opt }}(\bigcirc)$ for different values of $\gamma$ from 0 to 0.1 on the Poincaré sphere. Arrows indicate increasing values of $\gamma$. 

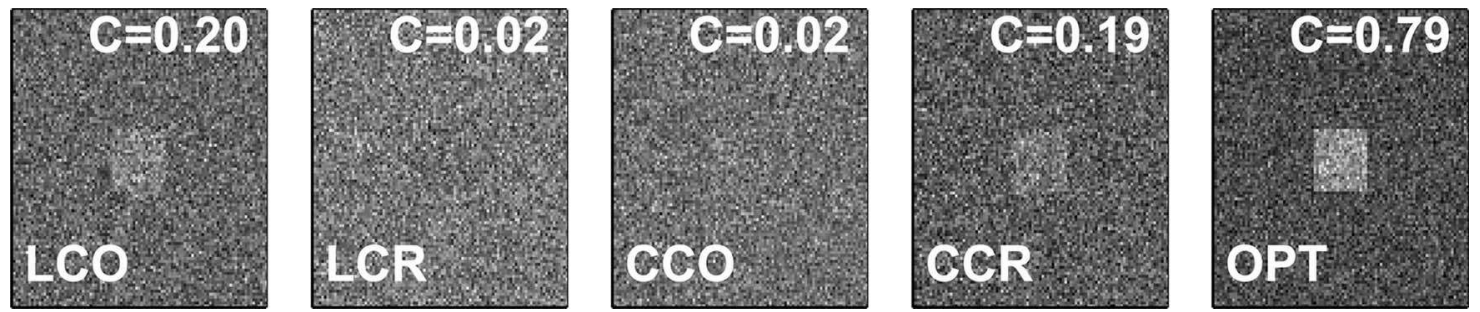

Fig. 4. Simulated images of a square piece of material $\left(M_{a}\right.$ with $\left.\gamma=0.096\right)$ on a background $\left(M_{b}\right)$ with $I_{0}=30, \sigma=10$. Each configuration is detailed in Table 1 .

terms is maximized. Finally, if $D_{00}=0$, that is, regions $a$ and $b$ have the same unpolarized intensity reflectivity, and thus their contrast is only due to polarimetric properties, both signs of $\vec{t}_{\text {opt }}$ lead to the same optimal value.

Let us now consider the case where $\widetilde{D}=0$, that is, the two regions differ only by their polarization and/or their diattenuation vectors. Equation (5) shows immediately that if $D_{00}>0$, then $\vec{s}_{\text {opt }}=\vec{m} /\|\vec{m}\|$ and $\vec{t}_{\text {opt }}=\vec{n} /\|\vec{n}\|$, and if $D_{00}<0$, then $\vec{s}_{\text {opt }}=-\vec{m} /\|\vec{m}\|$ and $\vec{t}_{\text {opt }}=-\vec{n} /\|\vec{n}\|$. Here again, it is noticed that the polarimetric contribution to contrast must have the same sign as the intensity contribution.

Let us now consider a more general case where two regions have the following Mueller matrices:

$$
M_{a}=\left[\begin{array}{cccc}
1 & 0 & 0 & \gamma \\
0 & 0.5 & 0 & 0 \\
4 \gamma & 0 & 0.3 & 0 \\
-\gamma & 0 & 0 & 0.4
\end{array}\right],
$$

where $\gamma$ varies between 0 and 0.1 and $M_{b}$ $=\operatorname{diag}(0.9,0.3,0.3,0.4)$. We verified that all matrices are physical Mueller matrices [7]. When $\gamma=0$, we are in the first case considered above, that is $\vec{m}=\vec{n}=0$. As $D_{00}>0, \vec{s}_{\text {opt }}=\vec{t}_{\text {opt }}=(1,0,0)^{T}$, which corresponds to a linear horizontal state of polarization. When $\gamma$ reaches its maximum value of 0.1 , one tends toward the second case, that is $\widetilde{D} \approx 0$. In this situation, one obtains $\quad \vec{s}_{\text {opt }} \approx \vec{m}=(0,0,1)^{T} \quad$ and $\quad \vec{t}_{\text {opt }} \approx \vec{n} /\|n\|$ $=(0,0.97,-0.24)^{T}$. For $0<\gamma<0.1$ we use the previously described algorithm to find the value of $\vec{u}_{\text {opt }}$ that maximizes $G(\vec{u})$. We show in Fig. 2 the value of the reduced contrast function $G^{\prime}\left(\alpha_{s}, \varepsilon_{s}\right)=G(\vec{u})$ for $\gamma$ $=0.096$, where $\alpha_{s}$ is the azimuth and $\varepsilon_{s}$ the

Table 1. Configurations of $\vec{s}=\left(\cos 2 \alpha_{s} \cos 2 \varepsilon_{s}, \sin 2 \alpha_{s} \cos 2 \varepsilon_{s}, \sin 2 \varepsilon_{s}\right)^{T}$ and $\vec{t}=\left(\cos 2 \alpha_{t} \cos 2 \varepsilon_{t}, \sin 2 \alpha_{t} \cos 2 \varepsilon_{t}, \sin 2 \varepsilon_{t}\right)^{T}$ considered in Fig. 4

\begin{tabular}{ccccc}
\hline & $\alpha_{\vec{s}}$ & $\varepsilon_{\vec{s}}$ & $\alpha_{\vec{t}}$ & $\varepsilon_{\vec{t}}$ \\
\hline LCO & $0^{\circ}$ & $0^{\circ}$ & $0^{\circ}$ & $0^{\circ}$ \\
LCR & $0^{\circ}$ & $0^{\circ}$ & $90^{\circ}$ & $0^{\circ}$ \\
CCO & $0^{\circ}$ & $45^{\circ}$ & $0^{\circ}$ & $45^{\circ}$ \\
CCR & $0^{\circ}$ & $45^{\circ}$ & $0^{\circ}$ & $-45^{\circ}$ \\
OPT & $0^{\circ}$ & $36.9^{\circ}$ & $40.1^{\circ}$ & $-6.9^{\circ}$ \\
\hline
\end{tabular}

ellipticity of the Stokes vector $\vec{S}$ $=\left(1, \cos 2 \alpha_{s} \cos 2 \varepsilon_{s}, \sin 2 \alpha_{s} \cos 2 \varepsilon_{s}, \sin 2 \varepsilon_{s}\right)^{T}$. The maximum is reached for $\alpha_{s}=0^{\circ}$ and $\varepsilon_{s}=36.9^{\circ}$. In Fig. 3 we represent the evolution of $\vec{s}_{\text {opt }}$ and $\vec{t}_{\text {opt }}$ as a function of the parameter $\gamma$. At the beginning, that is $\gamma$ $=0$, they are identical, then $\vec{s}_{\text {opt }}$ evolves toward a lefthanded circular polarization, whereas $\vec{t}_{\text {opt }}$ tends toward a right-handed, slightly elliptical state whose azimuth is around $45^{\circ}$.

To illustrate these results, we have simulated in Fig. 4 images of a square piece of material (characterized by $M_{a}$ with $\gamma=0.096$ ) on a background (characterized by $M_{b}$ ). We compare different configurations (see Table 1): linear copolarized (LCO), linear cross-polarized (LCR), circular copolarized (CCO), circular cross-polarized (CCR), and optimized (OPT). We also compute the contrast for each situation with Eq. (2). It is clearly seen that the optimized configuration shows the best contrast (more than four times higher than the others).

In summary, a method has been given to compute the illumination and analysis polarization states that optimize the contrast for region discrimination in active polarimetric scalar images perturbed with additive Gaussian noise. A significant increase of the contrast can be obtained by adapting these states of polarization to the two regions to discriminate. The study of contrast optimization in the presence of other types of noise, such as photon detection noise [8], is an interesting perspective to this work, since the optimal polarization states may be different in this case.

The authors would like to thank the anonymous reviewers for comments and suggestions for improvement that greatly improved the manuscript.

\section{References}

1. A. B. Kostinski and W. M. Boerner, IEEE Trans. Antennas Propag. 35, 988 (1987).

2. A. A. Swartz, H. A. Yueh, J. A. Kong, L. M. Novak, and R. T. Shin, J. Geophys. Res. 93, 15252 (1988).

3. M. Floc'h, G. Le Brun, C. Kieleck, J. Cariou, and J. Lotrian, Pure Appl. Opt. 7, 1327 (1998).

4. D. Goldstein, Polarized Light, 2nd ed. (Marcel Dekker, 2003).

5. F. Goudail, P. Réfrégier, and G. Delyon, J. Opt. Soc. Am. A 21, 1231 (2004).

6. G. Golub and W. Kahan, SIAM (Soc. Ind. Appl. Math.) J. Numer. Anal. 2, 205 (1965).

7. R. S. Cloude and E. Pottier, Opt. Eng. 34, 1599 (1995).

8. V. L. Gamiz and J. F. Belsher, Opt. Eng. 41, 973 (2002). 